\title{
HEMISFERECTOMIA FUNCIONAL PRECOCE NA HEMIMEGALENCEFALIA ASSOCIADA À EPILEPSIA REFRATÁRIA
}

\author{
Luiz Fernando Fonseca ${ }^{1}$, Renato Pacheco de Melo², Arthur Cukiert $^{3}$, \\ Jose Augusto Burattini ${ }^{5}$, Pedro Paulo Mariani ${ }^{5}$, Ródio Brandão ${ }^{4}$, Lauro Ceda ${ }^{4}$, \\ Cristine Mella Baldauf ${ }^{5}$, Meire Argentoni ${ }^{5}$, Cássio Forster ${ }^{5}$, Carla Baise ${ }^{4}$
}

\begin{abstract}
RESUMO - A hemimegalencefalia (HME) é malformação congênita cerebral rara de etiologia desconhecida que pode se apresentar com síndrome epiléptica de inídio precoce e resistente à terapia com anticonvulsivantes, associada a comprometimento significativo do desenvolvimento neuropsicomotor. A hemisferectomia funcional (HF) tem-se mostrado alternativa eficaz nos casos refratários à terapêutica medicamentosa. Em número diminuto, crianças foram operadas antes dos seis meses de idade. Esse estudo relata duas crianças com idade inferior a 6 meses com HME e síndrome epiléptica catastrófica submetidasa HF e com boa evolução clínica.
\end{abstract}

PALAVRAS-CHAVE: hemimegalencefalia, hemisferectomia, epilepsia.

\begin{abstract}
Early functional hemispherectomy in hemimegalencephaly associated to refractory epilepsy
ABSTRACT - Hemimegalencephaly (HME) isa rare congenital brain malformation of unknown etiology. Patients with HME can present with an early onset epileptic syndrome which is often refractory to medical treatment and associated to impaired neurological development. Functional hemispherectomy (FH) has proven to be a valuable treatment alternative in patients with refractory epilepsy in this setting. Very few children operated under the age of 6 months and bearing HME and catastrophic epilepsy (CE) have been described in the literature. This study reports on 2 kids younger than 6 monthswith HME and CE submitted to FH with good clinical outcome.
\end{abstract}

KEY WORDS: hemimegalencephaly, hemispherectomy, epilepsy.

A hemimegalencefalia (HME) é malformação congênita rara de etiologia desconhecida, que envolve a fase de proliferação e diferenciação neuronal durante o segundo e o quarto mês de gestação ${ }^{1}$. A manifestação clínica mais comum associada a essa condição é a síndrome epiléptica, muitas vezes de difícil controle medicamentoso e de início precoce, ocorrendo geralmente no período neonatal, com grave atraso do desenvolvimento neuropsicomotor, hemi paresia e hemianopsia². O diagnóstico baseiase em critérios clínicos e exames de imagem, e tornou-se maisobjetivo apóso advento da ressonância magnética (RM).O tratamento preconizado para crianças em quem há associação com síndrome e- piléptica catastrófica da infância é a hemisferectomia funcional (HF), que sofreu importante aprimoramento técnico nos últimos anos.

Este estudo descreve duas crianças menores de 6 meses com HME e epilepsia de evolução catastrófica tratadas por meio de HF.

\section{CASOS}

Paciente 1 - Dois meses de vida, masculino, foi inicialmente admitido no Centro Geral de Pediatria-FHEMIG (Belo Horizonte) com três dias de vida devido a crises epilépticas iniciadas nos primeiros cinco minutos após o nascimento. Encontrava-se em uso de fenobarbital (3 $\mathrm{mg} / \mathrm{kg} / \mathrm{dia}$ ) e sem controle das crises. Tratava-se do segundo filho (irmão saudável) e possuía diagnóstico de

\footnotetext{
Hospital Centro Geral de Pediatria- FHEMIG (Belo Horizonte MG, Brasil), Serviço de Cirurgia de Epilepsia do Hospital Brigadeiro (São Paulo SP, Brasil) e Clínica de Epilepsia de São Paulo (São Paulo SP, Brasil): ${ }^{1}$ Preceptor- Coordenador da Residência Médica de Neurologia Infantil do Hospital Centro Geral de Pediatria - FHEMIG M G Neurologista Infantil do Hospital Mater Dei e da Associação Mineira de Reabilitação (AMR); ${ }^{2}$ Residente em Neurologia Infantil pelo Hospital Centro Geral de Pediatria - FHEMIG MG; ${ }^{3}$ Chefe do Serviço de Neurologia e Neurocirurgia, Hospital Brigadeiro, São Paulo; ${ }^{4}$ Assistente do Serviço de Neurologia e Neurocirurgia, Hospital Brigadeiro, São Paulo; ${ }^{5}$ Assistente do Serviço de Neurologia e Neurocirurgia do Hospital Brigadeiro e da Clínica de Epilepsia de São Paulo.
}

Recebido 18 Março 2004, recebido na forma final 11 Junho 2004. Aceito 30 Julho 2004.

Dr. Arthur Cukiert - Rua Dr. Alceu de Campos Rodrigues 247/121 - 04544-000 São Paulo SP - Brasil. E-mail: acukiert@uol.com.br 
hidrocefalia por meio de ultrassonografia realizada no período pré-natal. O parto foi normal, a termo e sem intercorrências; osíndices de Apgar foram 7 e 8 . O peso ao nascimento foi $3520 \mathrm{~g}$ e o perímetro cefálico $35,5 \mathrm{~cm}$. Ospaisnão eram consangüíneose não havia história familiar de doença neurológica.

À admissão no Hospital Centro Geral de PediatriaFHEMIG encontrava-se em bom estado geral, hipoativo, pouco reativo, com pouco contato com o olhar, sem déficit motor focal, hipotônico, com reflexososteotendíneos normoativose simétricos, fundo de olho com atrofia óptica, e hipertrofia de toda hemiface direita (Fig 1). Várias crises epilépticas parciaissimplesmotorasà esquerda orolinguais, de extremidades e piscamento ocular, com desvio da comissura labial para a esquerda, foram observadas durante o exame. O eletrencefalograma (EEG) mostrou desorganização do ritmo de base e assimetria hemisférica. No hemisfério cerebral direito foram notadosparoxismos epileptiformestipo pontas, polipontase espículas difusas,

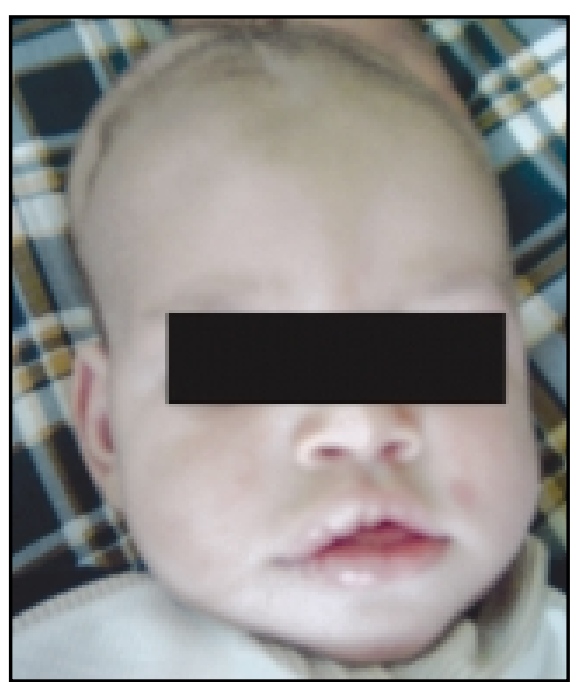

Fig 1. Fotografia mostrando hemihipertrofia de face direita no paciente 1. com eventual repercussão contralateral. A tomografia computadorizada (TC) mostrou assimetria hemisférica com aumento do volume do hemisfério cerebral direito associado à ventriculomegalia e diminuição de sulcose giros corticaise pouca diferenciação entre substância cinzenta e substância branca. A RM (Figs 2 e 3) mostrou acentuado aumento volumétrico do hemisfério cerebral direito com espessamento do manto cortical; áreas de polimicrogiria em lobo parietal e aspecto lisencefálico de córtex do lobo frontal. Presença de nódulo subependimário na superfície superior do ventrículo lateral direito com acentuada dilatação ventricular e adelgaçamento da calota craniana à direita. O SPECT ictal (Fig 4) mostrou hiperperfusão difusa de hemisfério cerebral direito, mais acentuada na região temporo- parietal.

Apóso diagnóstico, foi iniciado esquema anticonvulsivante que incluiu fenobarbital ( $5 \mathrm{mg} / \mathrm{kg} / \mathrm{dia})$, dobazam ( $1,5 \mathrm{mg} / \mathrm{kg} / \mathrm{dia}$ ) e topiramato ( $9 \mathrm{mg} / \mathrm{kg} / \mathrm{dia}$ ), sem controle satisfatório das crises epilépticas. A criança permaneceu

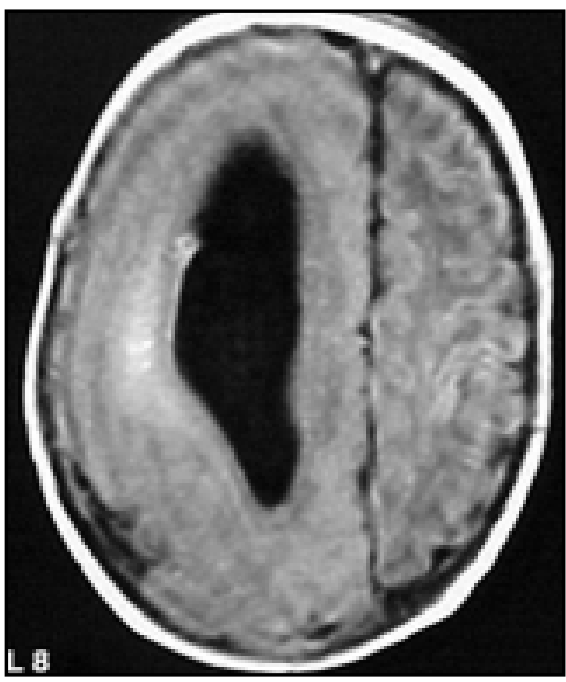

Fig 2. Corte axial de RM em FLAIR demonstrando a HME vista no paciente 1.
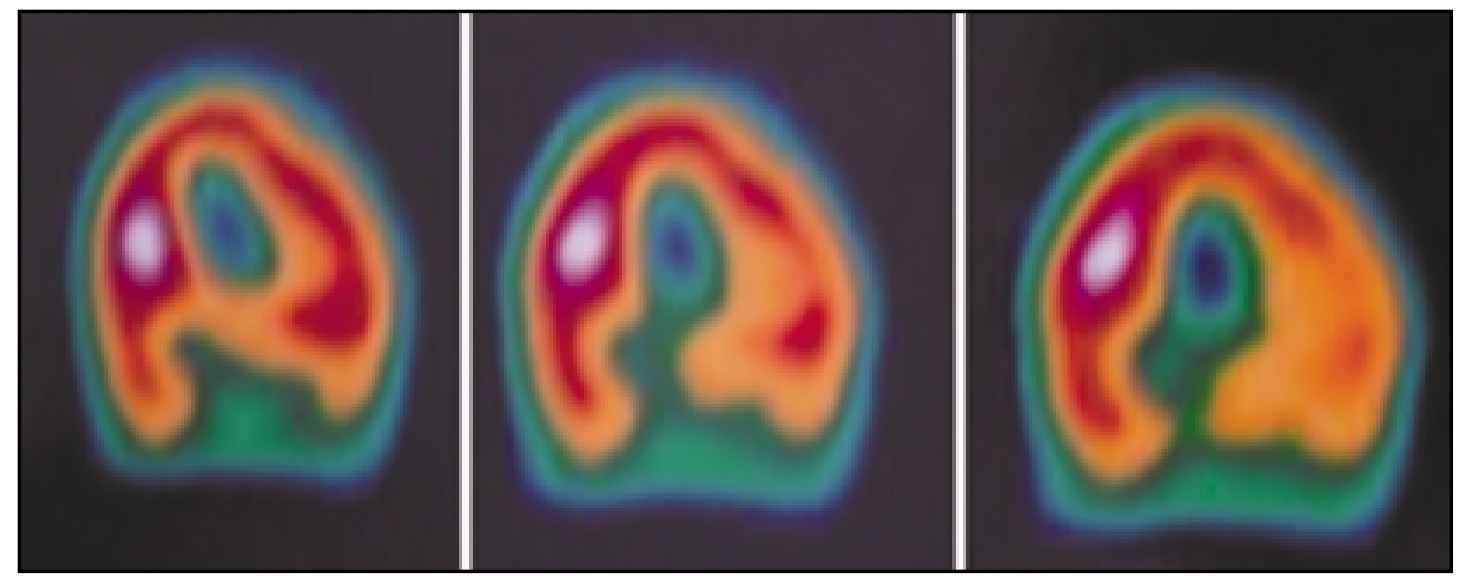

Fig 3. Cortes coronais de SPECT demonstrando hiperperfusão ictal associada ao hemisfério doente (paciente 1). 

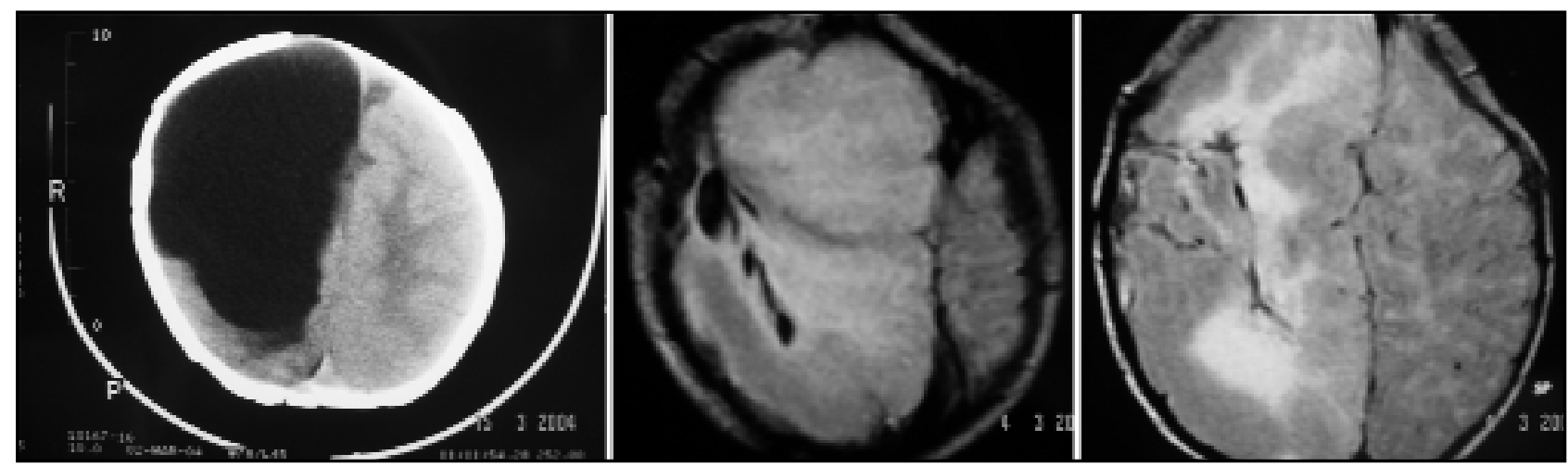

Fig 4. Paciente 1. Esquerda: corte axial alto de TC após a remoção da convexidade cortical; Centro: corte axial alto de RM em T1 pós-operatória mostrando remoção da convexidade fronto-parietal; Direita: corte axial mais baixo de RM em T1 pós-operatória, mostrando as desconexões corticais.

sempre internada, exceto por 3 dias de sua vida, na maioria das vezes em UTI, em status epilepticus. Com 2 meses de vida foi transferida ao Hospital Brigadeiro para ser submetido à HF. Encontrava-se na ocasião em mau estado geral, status epilepticus e com atraso no desenvolvimento neuropsicomotor (não sustentava a cabeça), sendo submetida à HF.

O procedimento consistiu da ressecção de extensas porções da convexidade fronto-parietal direita, lobectomia temporal anatômica, calosotomia e desconexão dos polosfrontal e occipital, que foram deixadosem posição.

Logo apóso procedimento permaneceu 10 diassem crises, com melhora progressiva do contato, melhora da hipotonia e da movimentação espontânea, apresentando discreto déficit motor à esquerda, que regrediu rapidamente. Apósesse período, crises parciais simples motoras diárias, na maioria das vezes restritas à face ,foram notadas, bem como generalizações eventuais. Atualmente, faz uso de oxcarbazepina ( $20 \mathrm{mg} / \mathrm{kg} / \mathrm{dia}$ ) e topiramato (5 mg/kg/dia). Está em acompanhamento fisioterápico, fonoaudiológico e de terapia ocupacional e em seguimento ambulatorial, não tendo sido mais internado por status após a cirurgia. Ainda apresenta retardo no desenvolvimento neuropsicomotor, porém com melhora progressiva; aos 11 meses de idade, sustenta a cabeça e iniciou controle postural de tronco.

Paciente 2 - Cinco meses de vida, masculino, apresentava crises parciais simplesmotorasà esquerda, versivas para a esquerda, com ruptura de atenção, e tônicas bilaterais desde a primeira semana de vida. Não havia antecedentes pessoaisou familiares. Não havia assimetria somática.

O EEG mostrou intensa atividade irritativa em hemisfério cerebral direito e sincronia bilateral secundária evidente. A RM mostrou HME à direita. O exame neurológico demonstrava atraso de desenvolvimento neuropsicomotor; aos 5 meses de vida encontrava-se hipotônico, não sustentava a cabeça e era freqüentemente internado em status epilepticus. Foi submetido aos 5 me- ses de vida à $\mathrm{HF}$, de modo similar ao Paciente 1; na ocasião encontrava-se em status epilepticus parcial.

No pós-operatório, houve melhora significativa do número das crises; persistem ainda crisesparciaissimples motoras diárias, com predomínio orofacial, eventualmente acometendo também o membro superior esquerdo. Crises tônicas bilaterais ocorrem na freqüência de 1 a 2 vezes por semana. Aos 8 meses de vida, a criança encontra-se em regime exclusivamente ambulatorial, não tendo mais sido internada por motivos neurológicos. Está iniciando agora a sustentação da cabeça.

Os pacientes foram tratados de acordo com o protocolo de cirurgia de epilepsia aprovados pelo Hospital Brigadeiro.

\section{DISCUSSÃO}

A HME foi descrita pela primeira vez por Sims em 1835 e caracteriza-se por proliferação neuronal excessiva com aumento de um hemisfério cerebral, dilatação e configuração irregular dos ventrículos e graus variáveis de displasia cortical, incluindo heterotopias, lisencefalia, polimicrogiria e anormalidades vasculares ${ }^{3-5}$. O mecanismo determinante desta malformação é desconhecido. Algunsautores sugerem que a agressão deve ocorrer no final do segundo trimestre de gestação, levando tanto à polimicrogiria quanto ao aumento de substância branca hemisférica devido a alterações de membrana celular e de receptores de moléculas que guiam o desenvolvimento axonal ${ }^{6}$. Outros autores divergem dessa teoria, sugerindo que um mosaicismo somático de células neuroectodérmicas primitivas pode ser o mecanismo fundamental.

Clinicamente, a HME pode ocorrer de forma isolada ou associada a outras alterações da pele como nevus sebáceo linear (síndrome de Jadassohn), angiomas cutâneos semelhantesaosencontradosnas 
síndromes de Sturge-Weber, hipomelanose de Ito, incontinência pigmentar, Klippel-Trenaunay-Weber. Em alguns pacientes evidencia-se hemi-hipertrofia somática coincidente com o lado do hemisfério cerebral acometido 7,8 .

O EEG pode ter valor preditivo quanto ao prognóstico. Complexos trifásicos de alta amplitude são observadosem casosmais graves, enquanto padrões de atividade alfa rítmica unilateral são visualizados em pacientes com prognóstico mais favorável ${ }^{9}$.

A exploração por meio de RM evidencia um hemisfério cerebral com aumento de seu volume, com efeito de massa que geralmente comprime o corpo do ventrículo lateral e acarreta desvio de linha média em sentido contralateral. Os giros são espessados, os sulcos pouco definidose a margem cortical interna indistinta. A substância branca apresenta-se com sinal hipo-intenso em Tl e hiperintenso em T2, sem captação anômala de contraste $^{10}$. Os métodos de neuroimagem funcional, tal como SPECT podem auxiliar no diagnóstico topográfico da região mais epileptogênica. A presença de foco epileptogênico no hemisfério de volume "normal" poderia comprometer o sucesso da hemis ferectomia.

A síndrome epiléptica presente na criança com HME apresenta variados graus quanto a sua gravidade. Ser refratária ao tratamento medicamentoso, o que freqüentemente ocorre na $\mathrm{HME}$, é a maior indicação para o tratamento cirúrgico. King et al, em 1985, foram os primeiros a descrever a hemisferectomia com resultado favorável realizada em um lactente de 5 meses de idade que obteve controle das crisese melhora do desenvolvimento neuropsicomotor apósa cirurgia ${ }^{11}$. Posteriormente, em 1989, Vigevano et al reportaram os resultados favoráveis de duas crianças com HME submetidas a hemisferectomia ${ }^{12}$.

A HF tem sido o tratamento cirúrgico preconizado, com índice reduzido de complicações advindas da técnica operatória, taiscomo hemossiderose, hidrocefalia , hematomas intracranianos, meningite pós-operatória e osteomielite. A HF tem produzido melhores resultados clínicos, quando comparada à hemisferectomia anatômica. Nesse procedimento, os polosfrontal e occipital são deixados in situ com suprimento sangüíneo intacto, mas são desconectados do restante do parênquima cerebral. Todo o resto da corticalidade é removido. Todas as fibras provenientes do lobo frontal e oc- cipital que se dirigem para o corpo caloso são interrompidas ${ }^{13-15}$.

O estudo anátomo- patológico revela neurônios gigantes ou outras características bizarras, grandes heterotopiasneuronaise perda da definição da junção do córtex com a substância branca, além de extensão variável de astrocitose ${ }^{16-18}$.

Os pacientes com HME já são funcionalmente hemisferectomizados, pois essa malformação impede o desenvolvimento das funções corticais superiores relacionadas ao hemisfério envolvido. Do ponto de vista funcional, esses pacientes desenvolvem-se com um único hemisfério cerebral, cujo funcionamento é ainda prejudicado pela atividade epileptogênica difusa contralateral.

A idade ideal para a realização da hemisferectomia ainda é assunto controverso e existem pouquíssimos pacientes descritos na literatura que foram submetidos à cirurgia antes dos 6 meses de vida, como é o caso dos pacientes aqui descritos. Alie-se o fato que nossos pacientes foram operados em péssima condição clínica e neurológica (status). De qualquer forma, é possível compreender osbenefícios da intervenção precoce caso ocorra controle dascrises epilépticas, possibilitando ganhosno desenvolvimento neuropsicomotor e melhor integração social da criança no futuro. Em sua maioria, crianças portadoras de HME associada à epilepsia catastrófica precoce que não foram submetidas à hemisferectomia falecem antes dos dois anos de vida (comunicação pessoal, A Cukiert) ou possuem profundo retardo de desenvolvimento.

As displasias corticais extensas representam substrato relacionado a pior prognóstico cirúrgico em crianças submetidas à $\mathrm{HF}$, em especial quando comparadas àquelas portadoras de síndromes hemiplégicas ou de Rasmussen. Se bem que nas crianças com HME seja óbvio que a patologia mais intensa encontra-se no hemisfério maior, existem fortes evidências de que essas criançastambém pos suam anormalidades no hemisfério considerado, inicialmente, como são. Isso encontra-se de acordo como retardo de desenvolvimento neuropsicomotor de muitas delas mesmo após cirurgia bem sucedida no que diz respeito às crises. Além disso, crises residuais podem estar relacionadas ao hemisfério considerado normal. As crises residuais após HF relacionam-se, no entanto, maisfreqüentemente a restos de parênquima anormal que não foram adequadamente desconectados. Crises parciais motoras com predomínio orofacial, como vistas 
no pós-operatório de nossas crianças, nas quais o córtex motor foi totalmente removido, podem ter origem no cortex insular, que é pobremente desconectado na HF, em especial em sua porção mais caudal.

O procedimento de desconexão é especialmente difícil nas criançascom HME, já que elas, apesar de possuírem hemisférios grandes, mostram sistema ventricular freqüentemente distorcido e malformado e, em alguns casos, inexistente. Como a desconexão é em geral realizada tomando por pontos de reparo aspectos topográficos intraventriculares, que se encontram distorcidos, o cirurgião acaba tendo que utilizar outros métodos de localização intraoperatória durante o procedimento, em especial, a comparação direta entre a anatomia cortical e aspectos topográficos avaliados na RM.

Apesar de representar uma solução agressiva, a HF precoce parece ser, no momento, a melhor forma de tratamento dessa forma de epilepsia catastrófica da infância.

\section{REFERÊNCIAS}

1. Piovesana AMSG, Cendes F. Malformações do SNC e desordens do desenvolvimento cortical. In Fonseca LF, Pianetti G, Xavier CC (eds), "Compêndio de Neurologia Infantil" Belo Horizonte MEDSI, 2002:219-236.

2. Di Rocco C, Iannelli A, Marchese E, Vigevano F, Rossi GF. Terapia chirurgica dell emimegalencefalia epilleptogena. Minerva Pediatr 1994;46:231-237.
3. Sims JM. On hypertrophy and atrophy of the brain. Medicochir Trans 1835;19:315-380.

4. Yasha TC, Santosh V, Shankar SK. Hemimegalencephaly: morphological and immunocytochemical study. Appl Neuropathol 1997;16:17-22.

5. Bosman C, Boldrini R, Dimitri L, Di Rocco C, Corsi A. Hemimegalencephaly: histological, immunohistochemical, ultrastructural and cytofluorimetric study of six patients. Childs Nerv Syst 1996;12:765-775.

6. Barkovich AJ, Chuang SH. Unilateral megalencephaly: correlation of MR imaging and pathologic characteristics. Am J Neuroradiol 1990;11:523-531.

7. Bianchine JW. The nevous sebaceous of Jadassohn. Am J Dis Child 1970;120:223-228.

8. Cavenagh EC, Hart BL, Rose D. Association of linear sebaceous nevus syndrome and unilateral megalencephaly. AJNR 1993;14:405-408.

9. Paladin F, Chiron C, Dulac O. Electroencephalographic aspects of hemimegalencephaly. Dev Med Child Neurol 1989;31:377-383.

10. Renowden SA, Squier M. Unusual magnetic resonance and neuropathological findings in hemimegalencephaly: report of a case following hemispherectomy. Dev Med Child Neurol 1994;36:357-369.

11. King M, Stephenson JB, Ziervogel M. Hemimegalencephaly: a case of hemispherectomy? Neuropediatrics 1985;16:46-55.

12. Vigevano F, Bertini E, Boldrini R. Hemimegalencephaly and intractable epilepsy : benefits of hemispherectomy. Epilepsia 1989;30:833-843.

13 Smith SJM, Andermann F, Villemure JG. Functional hemispherectomy: EEG findings, spiking from isolated brain postoperatively, and prediction of outcome. Neurology 1991;41:1790-1794.

14. Villemure JG, Rasmussen T. Functional hemispherectomy in children. Neuropediatrics 1993;24:53-55.

15. Taugher B, Richards M. Functional hemispherectomy. Axone 1992;14:29-32.

16. Rosa MJD, Secor DL, Barsom M. Neuropathologic findings in surgically treated hemimegalencephaly: immunohistochemical, morphometric, and ultrastructural study. Acta Neuropathol 1992;84:250-260.

17. Takashima S, Chan F, Becker LE. Aberrant neuronal development in hemimegalencephaly: immunohitochemical and Golgi studies. Pediatr Neurol 1991;7:275-278.

18. Sarnat HB. Cerebral dysgenesis: embryology and clinical expression, New York; Oxford University Press, 1992. 